\title{
Micro-differential thermal analysis detection of adsorbed explosive molecules using microfabricated bridges
}

Senesac, Larry R.; Yi, Dechang; Greve, Anders; Hales, Jan Harry; Davis, Zachary James; Nicholson, Don M.; Boisen, Anja; Thundat, Thomas

Published in:

Review of Scientific Instruments

Link to article, DOI:

$10.1063 / 1.3090881$

Publication date:

2009

Document Version

Publisher's PDF, also known as Version of record

Link back to DTU Orbit

Citation (APA):

Senesac, L. R., Yi, D., Greve, A., Hales, J. H., Davis, Z. J., Nicholson, D. M., Boisen, A., \& Thundat, T. (2009). Micro-differential thermal analysis detection of adsorbed explosive molecules using microfabricated bridges. Review of Scientific Instruments, 80(3), 035102. https://doi.org/10.1063/1.3090881

\section{General rights}

Copyright and moral rights for the publications made accessible in the public portal are retained by the authors and/or other copyright owners and it is a condition of accessing publications that users recognise and abide by the legal requirements associated with these rights.

- Users may download and print one copy of any publication from the public portal for the purpose of private study or research.

- You may not further distribute the material or use it for any profit-making activity or commercial gain

- You may freely distribute the URL identifying the publication in the public portal 


\title{
Micro-differential thermal analysis detection of adsorbed explosive molecules using microfabricated bridges
}

\author{
Larry R. Senesac, ${ }^{1,2}$ Dechang Yi, ${ }_{3}^{1}$ Anders Greve, ${ }^{3}$ Jan $\mathrm{H}_{1}$. Hales, ${ }^{3}$ Zachary J. Davis, ${ }^{3}$ \\ Don M. Nicholson, ${ }^{1}$ Anja Boisen, ${ }^{3}$ and Thomas Thundat ${ }^{1,2, a)}$ \\ ${ }^{1}$ Oak Ridge National Laboratory, Oak Ridge, Tennessee 37831, USA \\ ${ }^{2}$ Department of Physics, University of Tennessee, Knoxville, Tennessee 37996, USA \\ ${ }^{3}$ MIC, Technical University of Denmark, Lyngby DK-2800, Denmark
}

(Received 25 November 2008; accepted 9 February 2009; published online 4 March 2009)

\begin{abstract}
Although micromechanical sensors enable chemical vapor sensing with unprecedented sensitivity using variations in mass and stress, obtaining chemical selectivity using the micromechanical response still remains as a crucial challenge. Chemoselectivity in vapor detection using immobilized selective layers that rely on weak chemical interactions provides only partial selectivity. Here we show that the very low thermal mass of micromechanical sensors can be used to produce unique responses that can be used for achieving chemical selectivity without losing sensitivity or reversibility. We demonstrate that this method is capable of differentiating explosive vapors from nonexplosives and is additionally capable of differentiating individual explosive vapors such as trinitrotoluene, pentaerythritol tetranitrate, and cyclotrimethylenetrinitromine. This method, based on a microfabricated bridge with a programmable heating rate, produces unique and reproducible thermal response patterns within $50 \mathrm{~ms}$ that are characteristic to classes of adsorbed explosive molecules. We demonstrate that this micro-differential thermal analysis technique can selectively detect explosives, providing a method for fast direct detection with a limit of detection of 600 $\times 10^{-12}$ g. () 2009 American Institute of Physics. [DOI: 10.1063/1.3090881]
\end{abstract}

\section{INTRODUCTION}

Combating the surge in explosives-based terrorism requires cost effective explosive sensors that are highly selective and extremely sensitive. However, real-time detection of explosives with high sensitivity and selectivity is a challenging task. ${ }^{1-3}$ Use of explosives sniffing dogs, though attractive, is not very practical. ${ }^{4,5}$ Presently used detection techniques are either bulky and expensive, such as ion mobility spectrometry devices, ${ }^{6}$ or suffer from low sensitivity. Despite their high selectivity, techniques based on Raman and laserinduced breakdown spectroscopies have weak signals making them better suited to bulk material detection than to trace vapor detection. $^{7}$ Therefore, at present there exists an urgent need for developing explosive trace sensors that satisfy requirements such as high selectivity, high sensitivity, realtime operation, low cost, and low power consumption. Recently developed sensors based on microfabricated microelectromechanical systems (MEMS) cantilever sensors can potentially satisfy all the requirements for an ideal explosive trace sensor, except for selectivity. The MEMS sensors, such as microfabricated cantilever beams, are physical sensors capable of detecting extremely small changes in displacements (forces) and mass, but do not offer any intrinsic chemical selectivity for molecular recognition. When used as a chemical sensor they measure the changes in force induced by molecular adsorption or mass loading due to adsorption. Chemical selectivity in MEMS sensors is often accomplished

\footnotetext{
${ }^{\text {a) }}$ Author to whom correspondence should be addressed.
}

by using chemically selective interfaces ${ }^{8}$ immobilized on their surfaces. Therefore, the selectivity of detection in these MEMS sensors is directly related to the selectivity of the interaction between the analyte molecules and the immobilized selective layers. Selective layers capable of reversible interaction with the analyte molecules are, however, very unspecific since they rely on generic weak interactions such as hydrogen bonding.

One way of overcoming this selectivity challenge is by incorporating a separation technique prior to detection. Disadvantages of this approach, however, are increased detection time and integration challenges involved with incorporating separation techniques with the miniature sensor platform. Another way of achieving selectivity, for example, for cantilever sensors, is by using cantilever arrays where each cantilever in the array is modified with a partially selective chemical layer. The response of the array is then analyzed with a pattern recognition algorithm. ${ }^{9-13}$ There are only a limited number of weak reversible chemical interactions that can serve as a basis for designing the selective layers. This small number of reversible interactions limits the number of orthogonal basis vectors that are required by pattern recognition algorithms to produce unique recognition selectivity. The response from an array, despite the number of elements in the array, will, therefore, be ratio metric without providing any selectivity. The molecular recognition interfaces based on weak interactions are not specific enough to produce unique responses with a single sensor. Therefore, despite all the advantages offered by MEMS sensors as a platform for the ideal chemical sensor, their use as a practical 
sensor requires developing techniques by which adsorptioninduced orthogonal signals can be easily extracted.

Recently we demonstrated that MEMS bridges can be used for obtaining signals that are orthogonal to those produced by coating-based approaches by utilizing one of the most underexploited properties of MEMS sensors: their extremely low thermal mass. ${ }^{14}$ It has been demonstrated that the low thermal mass of MEMS bridges allows controllable sensor heating rates, $d T / d t$, where $T$ is the temperature and $t$ is the time, of up to $10^{8}{ }^{\circ} \mathrm{C} / \mathrm{s} .{ }^{15}$ Here we show that unique thermal characteristics of adsorbed analytes can be obtained by controlled heating of microfabricated bridges with surface adsorbed analyte and that these thermal response curves agree very well with theoretical response patterns based on a combination of melting, evaporation, sublimation, desorption, and exothermic deflagration.

Current thermal material analysis techniques include differential scanning calorimetry (DSC), differential thermal analysis (DTA), temperature programmed desorption (TPD), and thermogravimetric analysis (TGA). In DSC the amount of heat required to raise the temperature of a sample is compared to a reference sample that has a well defined heat capacity over the range of temperatures to be scanned. Both sample and reference are maintained at the same temperature while the temperatures of both are slowly raised and the difference in the amount of heat needed to increase the temperature of the sample as compared to the reference is plotted as a function of temperature. This process is good for studying phase transitions in the sample such as melting and exothermic decomposition. DTA is a process very similar to DSC except that it is the heat flow into the sample and reference which is kept constant and the differential temperature between the sample and reference is measured and plotted as a function of time, or as a function of the reference temperature. TPD is a useful technique for observing desorption of molecules from a surface. In TPD the sample material adsorbed on a surface is heated and a mass spectrometer is used to detect and analyze the molecules as they desorb and the corresponding desorption temperature is recorded. The last method (TGA) measures very small changes in weight in relation to changes in temperature. It is useful for determining the decomposition points of explosives.

Our technique is most like DTA with the added sensitivity to desorption as in TPD. However where DTA requires relatively large samples (1-100 mg) and long analysis times (minutes to hours), our method can detect picograms in microseconds. The small thermal mass of our micromachined detector and the ultrasmall mass of the sample allow for very rapid heating cycles. As with TPD, our method can take advantage of a programmable heating rate to increase the device sensitivity to specific thermal characteristics of interest. Experiments with thermally induced deflagration of adsorbed explosives on microcantilevers ${ }^{16,17}$ were able to differentiate energetic materials from nonenergetic materials, however, they were not able to differentiate between the individual explosives. In addition to identifying energetic material, the micro-differential thermal analysis (DTA) approach presented here allows the identification of different explosives. This technique offers an unprecedented opportunity to distin-
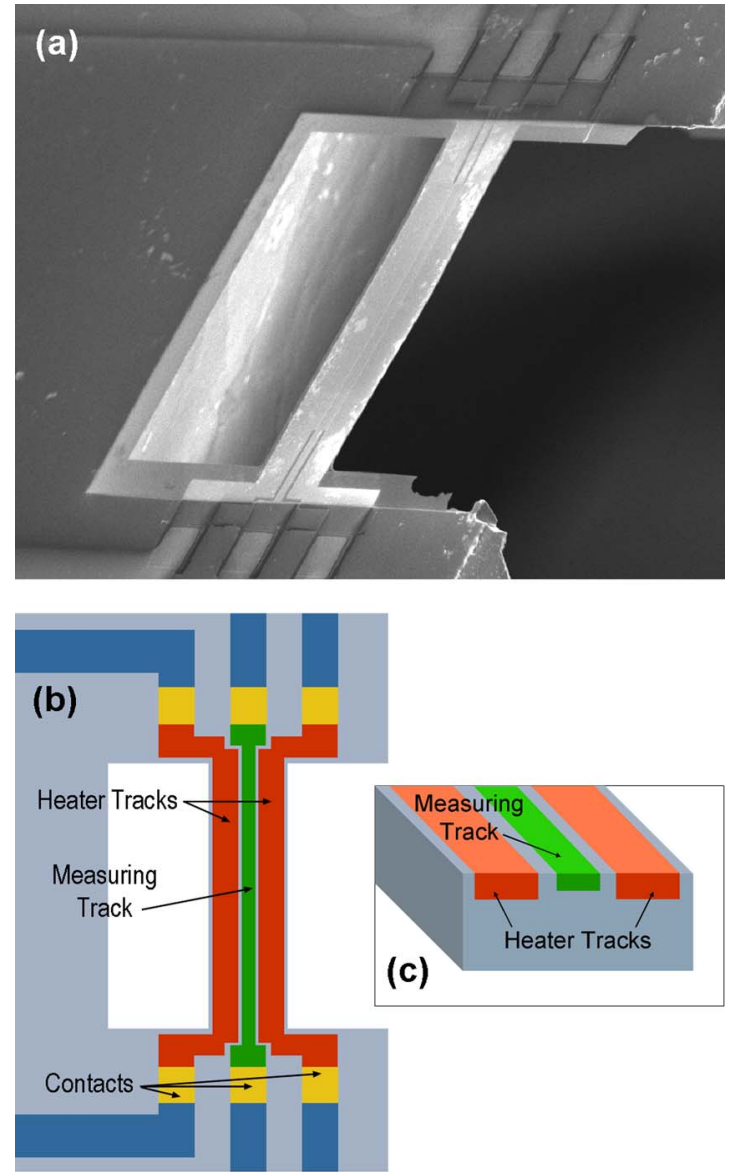

FIG. 1. (Color online) Bridge sensor structure. (a) A SEM image of a microfabricated silicon bridge structure with dimensions of $500 \mu \mathrm{m}$ in length, $100 \mu \mathrm{m}$ in width, and $550 \mathrm{~nm}$ in thickness. Imbedded in the bridge are two heater tracks, one on either side of a resistive temperature measuring track in the middle as shown in (b) and (c).

guish subnanogram quantities of adsorbed material without relying on chemoselective layers or separation methods.

\section{EXPERIMENTATION}

Our sensor, resembling a suspended bridge [Fig. 1(a)], was microfabricated from silicon-on-insulator (SOI) wafers with three buried electrically conducting tracks in each bridge with all tracks separated by thin layers of insulating silicon rich nitride [Figs. 1(b) and 1(c)]. There are three buried conducting tracks on each bridge. The middle track is used as a resistive temperature-measuring strip while the two outside tracks are used for heating the bridge by passing electrical current through them.

Figure 1(a) shows a scanning electron microscopy (SEM) image of the microfabricated suspended bridge. The bridge structures with heating tracks were fabricated on a SOI wafer with $400 \mathrm{~nm}$ buried oxide and $340 \mathrm{~nm}$ device layer. First the device layer is thinned to $200 \mathrm{~nm}$ by dry thermal oxidation, followed by boron doping by ion implantation to an active level of $\left(7 \times 10^{19} \mathrm{~cm}^{-3}\right)$. The resistors are defined by a reactive ion etch (RIE) step. A $250 \mathrm{~nm}$ layer of low pressure chemical vapor deposition (LPCVD) silicon rich nitride was deposited to provide electrical insulation between the resistors and to support the structure. A $90 \mathrm{~nm}$ 
layer of LPCVD polysilicon was deposited. The bridge and contact holes were defined using RIE and nitride etch (phosphoric acid at $180^{\circ} \mathrm{C}$ ). The nitride on the backside was patterned by RIE and the structures were then released by a $\mathrm{KOH}$ etch at $80^{\circ} \mathrm{C}$. During the release the front side was mechanically protected. Finally a metal layer of Ti/Au was deposited and wires were defined by an etch sequence [KI, $\mathrm{I}_{2}$, and hydrofluoric acid (HF)]. The bridge was $500 \mu \mathrm{m}$ long and $100 \mu \mathrm{m}$ wide. The two heating resistor tracks were $35 \mu \mathrm{m}$ wide and the measuring track in the center of the bridge was $10 \mu \mathrm{m}$ wide. The heaters had a resistance of $1.77 \mathrm{k} \Omega$ and the measuring resistor was $4.96 \mathrm{k} \Omega$. A high doping level was chosen so that the bridge can be heated to higher temperature before the intrinsic doping concentration interferes with the signal. Further details regarding bridge fabrication will be published elsewhere.

When the bridge is heated by passing current through the outermost tracks, the resistance of the internal track varies sensitively as a function of temperature. It is possible to heat the bridge to hundreds of ${ }^{\circ} \mathrm{C}$ in a few microseconds using this arrangement. The rate of heating depends on the mass of the bridge. Therefore, a bridge with added mass (adsorbed mass) will have a different heating rate compared to a bare bridge. Since all the adsorbed mass leaves (desorption) by the end of the heating cycle, the response from a second heating step can act as a baseline signal representing the bridge with no adsorbed mass (Fig. 2). We used a Wheatstone bridge circuit to measure the difference in response between the adsorbed molecule loaded sensor MEMS bridge and an identical unloaded reference MEMS bridge.

During measurements, the sensor MEMS bridge was kept in the open air directly in the explosive vapor plume from a custom-made vapor generator with heated outlet. The explosive generators were developed at Idaho National Laboratory. They produce vapor containing explosive molecules by passing dry nitrogen through a reservoir containing an explosive standard heated to high temperature to increase the concentration of the explosive picked up by the gas. Further details of the vapor generators can be found elsewhere. ${ }^{18}$ These explosive vapors are delivered to the MEMS bridge and kept in open air using a heated outlet. The explosive vapor condenses on the MEMS bridge, which was maintained at room temperature. The identical reference MEMS bridge was protected from vapor adsorption. The explosives used were trinitrotoluene (TNT), pentaerythritol tetranitrate (PETN), and cyclotrimethylenetrinitromine (RDX).

The micro-DTA method described here is capable of providing unique signals for subnanogram quantities of adsorbed explosives within $50 \mathrm{~ms} .{ }^{14}$ This technique combines the phase transition detection capabilities of DTA with the temperature programming versatility of TPD, but carried out at a higher $d T / d t$ of $10^{4}{ }^{\circ} \mathrm{C} / \mathrm{s}$ (typical DTA rates are from .001 to $10^{\circ} \mathrm{C} / \mathrm{s}$ ). This rapid heating method opens up the possibility of investigating the thermal behavior, including thermally induced decomposition of subnanogram quantities of materials, in milliseconds. The differential signal measured as a function of heating time is proportional to the
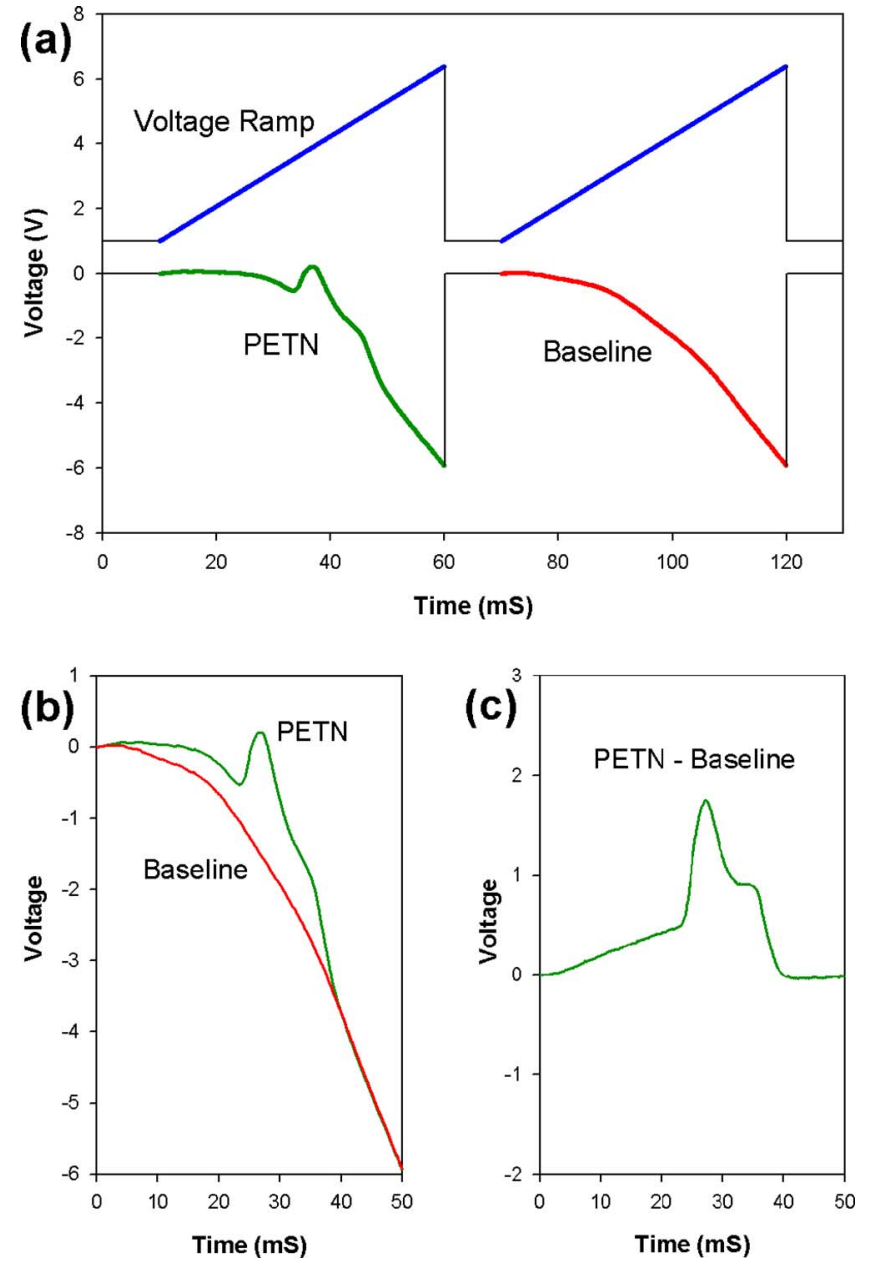

FIG. 2. (Color online) Signal timing and response. Two successive voltage ramps for heating are shown in (a). Each linear heating voltage ramp (0-5.4 $\mathrm{V}$ in $50 \mathrm{~ms}$, shown offset by $1 \mathrm{~V}$ for clarity) produces an average $d T / d t$ of $10^{4}{ }^{\circ} \mathrm{C} / \mathrm{s}$ on the MEMS bridges. The bottom curve in (a) shows the Wheatstone bridge circuit response, measured as a voltage, for two successive heating cycles. The Wheatstone bridge circuit output is the differential response between the analyte loaded sensor MEMS bridge and the unloaded reference MEMS bridge. The calibration for converting voltage to temperature difference between the two bridges is $3.75{ }^{\circ} \mathrm{C} / \mathrm{V}$. The first heating cycle with PETN adsorbed on the sensor bridge produces a different response than the second heating cycle because the PETN completely leaves the surface during the first heating cycle. The second cycle therefore produces a baseline signal indicating the normal heating difference between the loaded sensor bridge and the reference bridge caused by a small difference in characteristic temperature sensitivity between the two bridges. (c) The final response signature is produced by subtracting the baseline signal from (b) the signal with PETN present. The peak in the PETN signature corresponds to a maximum $6.6^{\circ} \mathrm{C}$ temperature difference between the two heating cycles, indicating that the sensor bridge was $6.6^{\circ} \mathrm{C}$ cooler (at peak) during the cycle with adsorbed PETN than it was during the unloaded cycle.

difference in resistance between the sensor bridge and the reference bridge, which is proportional to the difference in their temperatures. This measurement is also proportional to the rate of change in thermal mass of the sensor bridge due to thermal desorption of the adsorbed molecules $(d M / d T)$. Since the observed signal is with respect to a reference bridge, the mechanical buckling of the bridge under thermal stress does not play a role in the signal production. In our case, during heating, the adsorbed molecules undergo 
decomposition and desorption as a function of temperature. The mass loss rate $(d M / d t)$ is related to the product of $d M / d T$ and the heating rate $(d T / d t)$. The mass loss due to evaporation resembles a sigmoid function and the rate of evaporation resembles a Gaussian peak. ${ }^{19}$

\section{EXPERIMENTS}

\section{A. Thermal response: Explosives and nonexplosives}

Increasing the temperature of the bridge with a high heating rate, $d T / d t$, using a voltage ramp [Fig. 2(a)] results in a thermal response curve characteristic to the thermal properties of the adsorbed explosives when measured in common mode rejection with an identical unloaded reference bridge. The thermal response of a bridge with adsorbed mass is expected to be different from the reference bridge due to additional thermal mass. We experimentally determined that for a $d T / d t$ value of $1.1 \times 10^{4}{ }^{\circ} \mathrm{C} / \mathrm{s}$, the thermal responses showed unique response shapes that are characteristics to classes of explosives as shown in Fig. 3. The differential response is created by subtracting the signal of the analyte loaded bridge from the signal of the unloaded reference bridge and therefore indicates how far the temperature of the loaded bridge lags behind the temperature of the reference bridge [Figs. 2(b) and 2(c)]. Each explosive can be distinguished using the shape of the temperature response. The three separate line curves plotted for each explosive in Fig. 3(a) correspond to three separate experiments with varying amounts of explosive. Notice that the amplitude of the response varies as a function of the mass of adsorbed explosive, but the shape of the response remains constant for a given explosive. Figure 3(b) shows the response curves for two nonexplosives: ammonium chloride $\left(\mathrm{NH}_{4} \mathrm{Cl}\right)$ and sodium borate $\left(\mathrm{N}_{2} \mathrm{~B}_{4} \mathrm{O}_{7}\right)$. The response curves of the nonexplosives appear as simple Gaussian peaks characteristic of evaporation events, ${ }^{19}$ where the peak positions vary with the specific material as well as the amount of material adsorbed. The two separate line curves plotted for each analyte correspond to two separate experiments with varying mass of the analyte.

In Fig. 4(a) the response curves for explosives and nonexplosives are shown together for comparison. The response shape for each explosive is very distinct. It is expected that all the adsorbed material leaves the bridge at the end of the temperature cycle as evidenced by the lack of difference in thermal characteristics with respect to a reference bridge during the application of a second heating pulse [Fig. 2(a)]. Unlike the observed responses for nonexplosives, explosive responses tend to overshoot at the end of the response curve as shown in Fig. 4(b). This overshooting indicates that the sensor bridge has a higher temperature than the reference bridge, suggesting an exothermic process. The distinct response curves observed with explosives are due to a combination of melting, evaporation, and decomposition. The shape for TNT shows a single peak with a slow rise and fast fall. The fall in the response curve is directly related to the rate at which the bridge's temperature is increasing probably due to a combination of the expected exothermic decomposition of the explosive and a decrease in thermal mass due to (a)

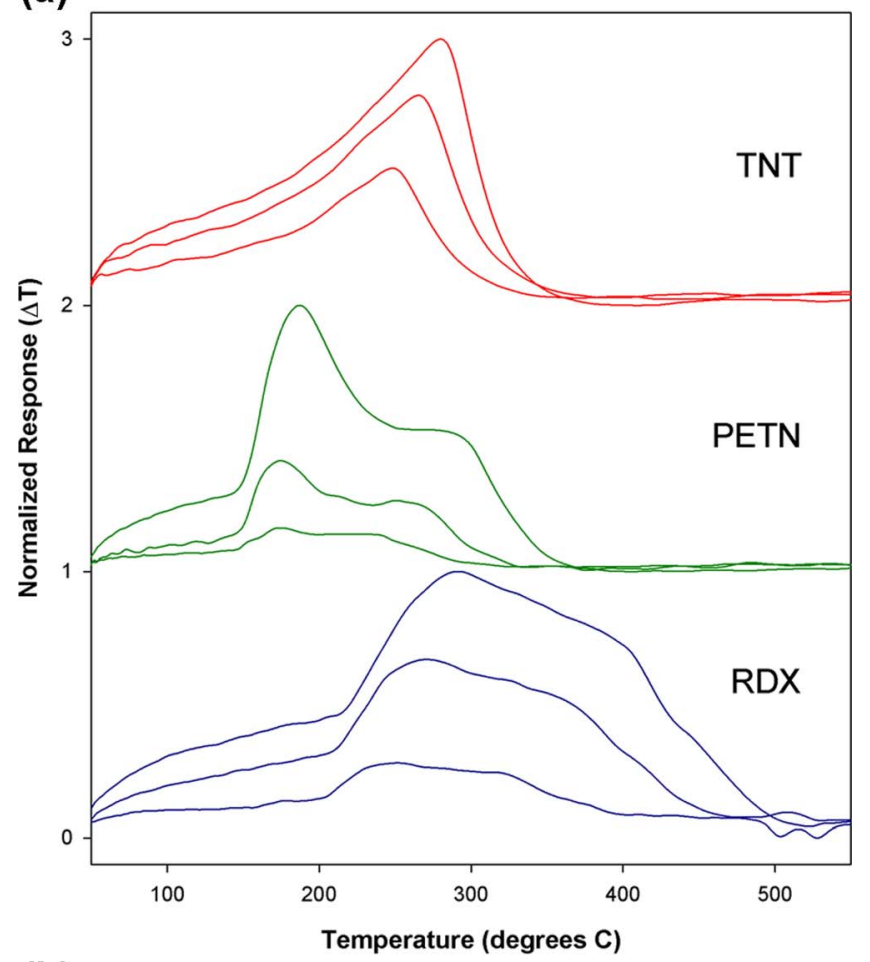

(b)

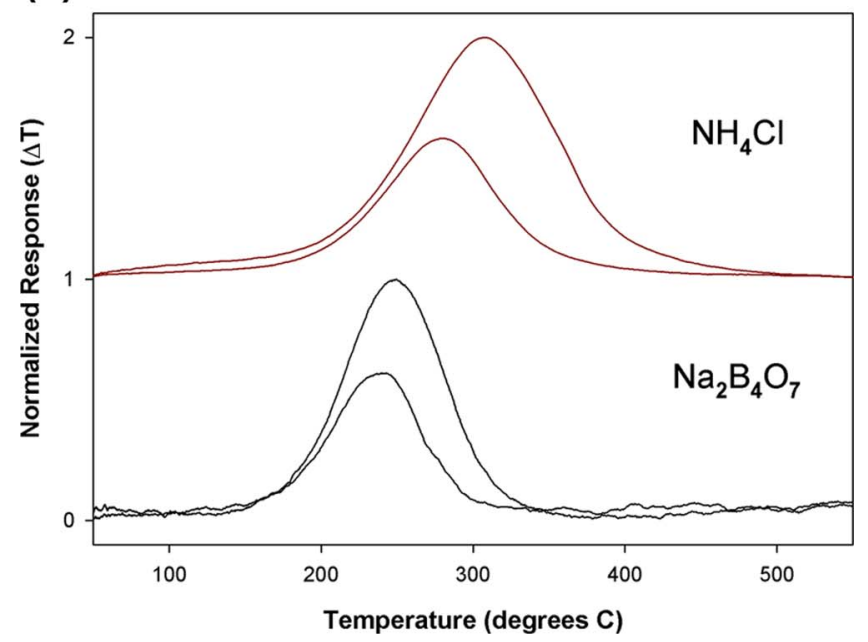

FIG. 3. (Color online) Response signatures of explosives and nonexplosives. (a) Thermal response of a bridge with adsorbed explosive molecules. Sensor output is plotted as a function of the reference bridge temperature ( $x$-axis) during heating. The response change in temperature $(\Delta T)$ is measured from changes in sensor bridge resistance with respect to a reference bridge such that a positive signal indicates that the temperature of the reference bridge is higher than that of the analyte loaded sensor bridge. Shown are the detector responses for three explosive species. The three different curves shown for each analyte represent $0.6,1.2$, and $2.4 \mathrm{ng}$ of adsorbed explosive on the device (mass determined by the method outlined in Sec. III C). (b) Thermal response of a bridge with adsorbed nonexplosive molecules carried out with the same $d T / d t$ as for the explosives. The thermal spectrum for nonexplosives shows a Gaussian response with peak value changing with the amount of adsorbate. Unlike the thermal spectrum for explosives, the nonexplosive spectra are featureless.

material leaving the surface. The slowly increasing shape is consistent with melting and evaporation of nucleated TNT on the surface as islands. Small islands evaporate faster than larger ones. 
(a)

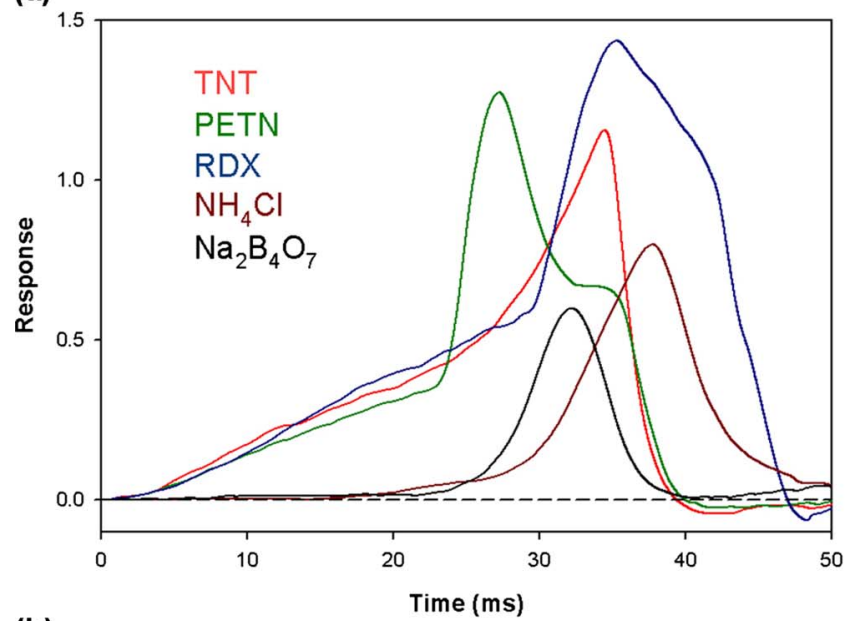

(b)

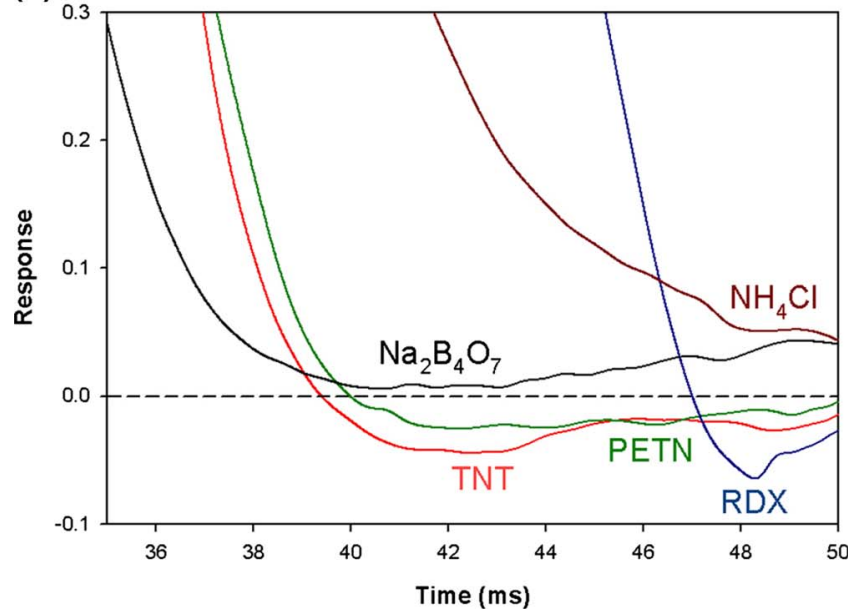

FIG. 4. (Color online) Thermal response shapes for explosives and nonexplosives. (a) Individual shapes for TNT, PETN, RDX, $\mathrm{NH}_{4} \mathrm{Cl}$, and $\mathrm{Na}_{2} \mathrm{~B}_{4} \mathrm{O}_{7}$ are plotted together here for ease of comparison. Nonexplosive responses show a Gaussian curve while explosive responses show distinct shapes due to a combination of melting, evaporation, and decomposition. Since the heating ramp voltage increases linearly with time and the temperature increases as the square of voltage (power goes as $\mathrm{V}^{2}$ ), the responses are plotted as a function of time to give a better sense (when compared to the temperature plot in Fig. 3) of how fast most of the transitions occur near the end of the pulse. (b) Close up of the region in (a) of observed overshooting where a signal below the dashed line indicates that the temperature of the bridge with adsorbed explosive is higher than the temperature of the bridge with no adsorbate present. This overshooting implies an exothermic decomposition. Note also that the nonexplosive curves do not fall below the dashed line indicating that the temperature of the bridge with adsorbed nonexplosive is always lower than the temperature of the bridge with no adsorbate present.

\section{B. Effect of nucleated structure of adsorbed explosives on desorption}

The atomic force microscopy (AFM) images of silicon oxide surfaces exposed to TNT vapors [Fig. 5(a)] show an island structure. The AFM images of nucleated islands as a function of time at room temperature show residues left behind, which are probably crystalline TNT. ${ }^{20}$ In our case, fast evaporation of islands leaves behind crystallites that exothermically decompose at higher temperature. The shapes of the thermal responses of RDX and PETN show a slow rise followed by a rapid rise to a distinct double peak and fast fall [Fig. 3(a)]. The initial slow rise can again be explained as
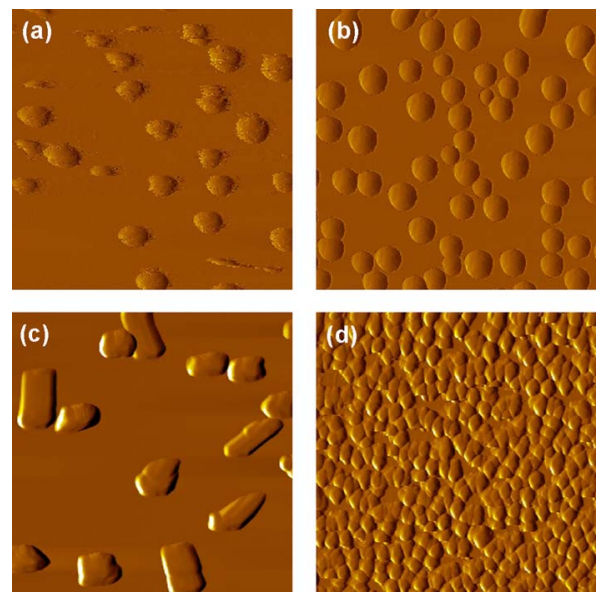

FIG. 5. (Color online) AFM images of adsorbed analyte on silicon oxide surface. AFM $\left(10 \times 10 \mu \mathrm{m}^{2}\right)$ images show nucleation islands of adsorbed (a) TNT, (b) PETN, and (c) RDX on a silicon oxide surface. The size of these islands increases with increasing exposure. (d) AFM image (10 $\times 10 \mu \mathrm{m}^{2}$ ) of vapor phase adsorption of $\mathrm{NH}_{4} \mathrm{Cl}$ on a silicon oxide surface shows uniform coverage with a finite surface roughness. The $\mathrm{NH}_{4} \mathrm{Cl}$ vapor was created by heating the $\mathrm{NH}_{4} \mathrm{Cl}$ in a small crucible placed under the silicon oxide surface. The height scale is $10 \mathrm{~nm}$ (from dark to light).

due to the evaporation of nucleated islands of explosives [Figs. 5(b) and 5(c)]. However, for PETN and RDX a rapid rise to a peak follows the slow rise at the melting point for each explosive $\left(141{ }^{\circ} \mathrm{C}\right.$ for PETN and $204{ }^{\circ} \mathrm{C}$ for RDX). This rapid rise to a peak may be due to the absorption of thermal energy needed to melt the PETN and RDX. The fall to the second peak followed by the fast fall is again related to the exothermic decomposition of the explosives coupled with a decrease in the thermal mass as in the case of TNT. The temperatures at which the response peaks occur were different for different explosives. The overshooting in the response curve as shown in Fig. 4(b) was observed only for explosives. A combination of characteristic shapes and overshooting due to an exothermic process can serve as a clear indicator for identification of classes of explosives. Compared to the explosives, the relatively simpler shape of the nonexplosives thermal spectrum indicates a pure evaporation from a uniform layer of adsorbed material. Unlike the explosives, the AFM images of $\mathrm{NH}_{4} \mathrm{Cl}$ show a uniform surface coverage [Fig. 5(d)]. Mass loss due to evaporation from a uniform surface coverage is expected to be a sigmoid and the rate of evaporation should resemble a Gaussian shape ${ }^{19}$ as observed in Fig. 3(b).

\section{Determination of mass concentration and limit of detection}

The mass of adsorbed explosive vapor was calculated using a resonating microcantilever beam placed in the same plane as the bridge sensor. For a cantilever free at one end, the frequency decreases as the adsorbed mass increases according to relation $\Delta m / m=-\Delta f / f$, where $m$ and $f$ represent the mass and resonant frequency of the cantilever, respectively. For our bridge structure the relationship is further complicated by the fact that both ends of the bridge are attached to the supporting substrate and therefore surface stress resulting from the adsorption of mass alters the spring con- 
stant of the device making it difficult to separate changes in resonant frequency due to mass loading (decreasing $f$ ) from changes due to surface stress (increasing $f$ ). The resonance frequency of the bridge structure was measured using an optical beam deflection method, which showed frequency increasing as a function of vapor adsorption due to adsorptioninduced surface stress effects. Therefore, the mass of explosive vapor adsorbed on the structure was estimated using a resonating reference cantilever device after exposure to the same duration of analyte vapor as was used for the bridge device. For calibration purposes, the mass adsorption was assumed to be uniform across the cantilever and the effective mass/area was calculated. It was also assumed that the mass/ area was the same for the bridge sensor and therefore the mass adsorbed on the bridge was calculated as the product of the cantilever adsorbed mass/area with the area of the bridge surface. From these resonance frequency measurements, the calculated limit of detection (LOD) for our device was approximately $0.6 \times 10^{-9} \mathrm{~g}$ of adsorbed mass, which is slightly less than the $1 \%$ of the inertial mass of the bridge and corresponds to $10^{11}$ molecules. Since the vapor pressures of explosives are extremely small, a preconcentrator is essential for collecting explosive molecules on the bridge surface. For example, the vapor pressure of PETN is around 5 ppt (volume) at room temperature, and, therefore, there are only $10^{14}$ molecules per $\mathrm{m}^{3}$ of air around PETN. However, the preconcentrators are designed for collecting explosive molecules and particulates by sampling large volumes of air. The collected molecules are then abruptly desorbed at which time the explosive concentrations can be as high as ppb or higher for detection. Work is presently underway to develop high efficiency preconcentrators that can collect low vapor pressure explosives. Explosives such as TNT have vapor pressure in the ppb range, and, therefore, may require only mild preconcentration.

\section{Interference from VOCs and humidity}

To illustrate high specificity in detection, we carried out experiments with interferents such as nonexplosives, volatile organic compounds (VOCs), and water vapor. Experiments conducted with VOCs and water vapor did not produce any discernable signals. This is probably due to the fact that the amount of mass adsorbed may be less than the detection threshold. Since the sticking coefficients of the VOCs are smaller than those of the explosives and the vapor pressures of the VOCs are higher, the mass loading of adsorbed VOCs is expected to be lower. One of the challenges in chemical vapor detection, especially explosive vapors, is the influence of relative humidity. Since the explosives vapor concentration is millions of times smaller than the water vapor concentration, relative humidity is a significant interferent. To determine the effect of relative humidity, we carried out experiments at different humidity levels. Even at $80 \%$ relative humidity, no thermal response signal was produced indicating that the system will work even in the presence of high humidity. It should be noted that direct exposure of the bridge surface to condensed water causes an electrical short circuit of the heating and measuring tracks. A thin coating of insulating material can be added to future fabrications to

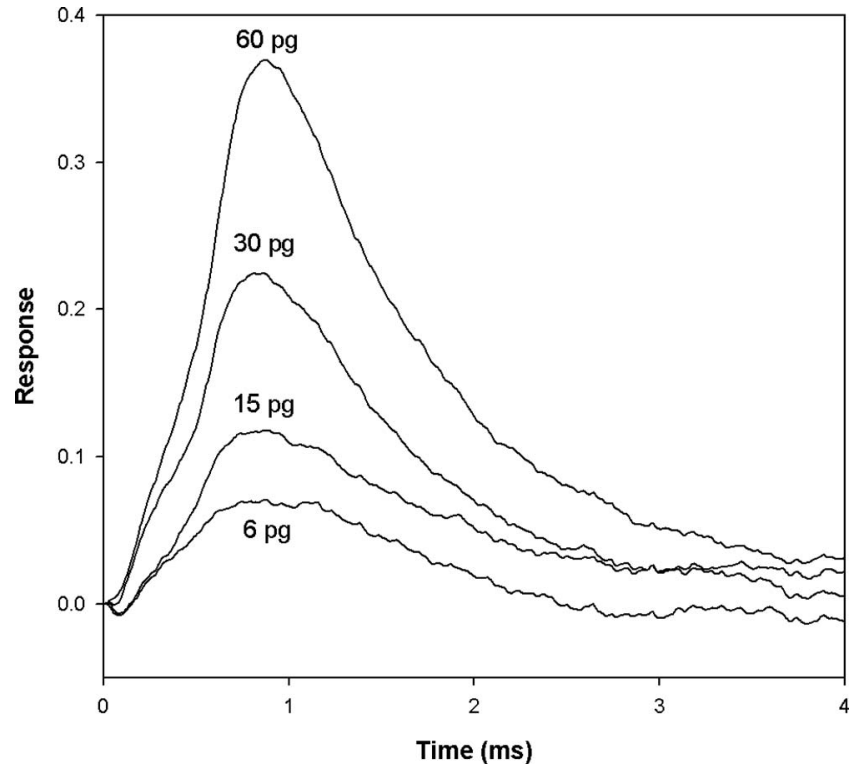

FIG. 6. Rapid pulse technique for higher detection sensitivity. Thermal response of a bridge with adsorbed TNT for rapid pulsed heating. A $20 \mathrm{~ms} 8$ $\mathrm{V}$ square wave pulse was used to heat the bridge. The pulse voltage and bridge temperature rise time is only about $50 \mu \mathrm{s}$. Only the first $4 \mathrm{~ms}$ of the data is shown here to give a better resolution examination of the response shapes. All the plots go to zero shortly after $4 \mathrm{~ms}$. The responses for adsorbed TNT do not show any features as observed with ramped heating. However, adsorbed TNT mass as low as 6 pg produced a response.

prevent this problem, however, it is expected that water condensed directly on the bridge surface would give a thermal mass signature response.

\section{E. Rapid heating rate leads to higher sensitivity}

The characteristic shapes of the thermal spectrums were significantly influenced by the value of $d T / d t$. At higher $d T / d t$ (square wave pulse with rise time to $550{ }^{\circ} \mathrm{C}$ in less than $50 \mu \mathrm{s}$ ), the observed peaks were merged into a single peak loosing their speciation characteristics. Figure 6 shows the thermal spectrums observed with TNT for fast heating. Similar unresolved spectrums were also observed for fast heating of RDX and PETN. Figure 6 shows that under fast heating, the peak temperature does not shift with the amount of explosive adsorbed. This is consistent with the evaporation rate of individual islands. The response is very unique to sticky molecules such as explosives, while VOCs and humidity do not show any observable thermal responses. However, the nonspecific response observed under fast heating has a LOD of $6 \mathrm{pg}$, which is a factor of 100 times smaller than what was found for our device with the slower $50 \mathrm{~ms}$ ramp heating. It is possible to combine pulsed and ramped heating approaches in such a way that ramped heating is initiated for speciation only if pulsed heating shows a response. A combined approach will result in a selective explosive sensor that is rapid, sensitive, and completely reversible.

\section{THERMODYNAMIC ANALYSIS}

The ramped heating method gives clear thermodynamic signatures that vary significantly with analyte and are 
therefore useful for analyte identification even without further interpretation of the thermodynamic information contained in the response curves. However, a virtue of the method with regard to signaling the presence of energetic molecules is that it is sensitive to molecular energies. An understanding of the response in terms of thermodynamics may lead to general identification of energetic materials even those whose signals have not been previously cataloged. Furthermore, thermodynamic understanding can guide the design of the bridge sensors so that selectivity can be optimized.

The unloaded bridge can be modeled by a total heat capacity, $C(\mathrm{~J} / \mathrm{K})$, an electrical conductivity $G\left(\mathrm{~J} / \mathrm{s} \mathrm{V}^{2}\right)$, and an average "cooling/heating" coefficient $k(\mathrm{~J} / \mathrm{K} \mathrm{s})$. We find that the unloaded bridge temperature is well described by the simple linear differential equation,

$$
C \frac{d \bar{T}}{d t}=G\left[V(t)^{2}-k\left(\bar{T}-T_{a}\right)\right],
$$

where $T_{a}$ is the ambient temperature $(300 \mathrm{~K})$ and $\bar{T}$ is the average temperature of the bridge. When the voltage is linear in time, i.e., $V(t)=r t$, the exact solution is

$$
\bar{T}-T_{a}=a\left[\left(\frac{t}{\tau}\right)^{2}-2\left(\frac{t}{\tau}\right)+2\left\{1-\exp \left[-\left(\frac{t}{\tau}\right)\right]\right\}\right],
$$

where $\tau=C / k$ and $a=\left(r^{2} G / k\right) \tau^{2}$. The linearity of Eq. (1) and the linearity of the thermistor (measuring track on bridge) justify the assumption that the thermistor temperature $T_{T}$ and the analyte temperature $T_{A}$ are proportional to the average bridge temperature $\bar{T}: T_{r}=\left(a_{T} / a\right) \bar{T}$ and $T_{A}=\left(a_{A} / a\right) \bar{T}$. Hence Eq. (2) also applies to the thermistor temperature $T_{T}$ or the average temperature of the analyte $T_{A}$. The function $\bar{T}(t)$ is simply multiplied by $a_{T} / a$ or $a_{A} / a$ to obtain $T_{T}$ and $T_{A}$, respectively. Fitting the two constants, $a_{T}$ and $\tau$, to the $T_{T}(t)$ of the unloaded bridge gives $a_{T}=1.7 \mathrm{~K}$ and $\tau=2.6 \mathrm{~ms}$. Note that the time constant $\tau$ of the bridge is a robust quantity that describes the exponential decay of temperature excursions $\left(\bar{T}, T_{T}\right.$, or $\left.T_{A}\right)$ back to the ambient temperature.

Next consider adsorbed analyte droplets on the bridge within a temperature range where the only transformation is to the vapor (evaporation or sublimation). We will use the term vaporization to indicate either sublimation or evaporation. Equation (1) is modified by the cooling due to the loss of analyte mass to vapor,

$$
C \frac{d \bar{T}}{d t}=G[V(t)]^{2}-k\left(\bar{T}-T_{a}\right)+\varepsilon_{V} \frac{d m}{d t},
$$

where, $\varepsilon_{V}$ is the enthalpy of vaporization. Taking the difference between Eq. (1) and Eq. (3) gives

$$
\begin{aligned}
& C \frac{d \bar{T}_{m_{0}}-\bar{T}_{0}}{d t}=-k\left(\bar{T}_{m_{0}}-\bar{T}_{0}\right)+\varepsilon_{V} \frac{d m}{d t}, \\
& C \frac{d \Delta T}{d t}=-k(m) \Delta T+\varepsilon_{V} \frac{d m}{d t} .
\end{aligned}
$$

In Eq. (4) a cancellation of terms is achieved between the loaded and unloaded bridge that is similar to the cancellation occurring in the experiment. In Eq. (4) the heat flow that is controlled by $k$ now returns $\Delta T$ to zero. By comparing the solution of Eq. (4) to our measurements, we find that more realistic values are obtained if we assume that the coating of the analyte on the surface reduces the coefficient $k$. We postulate that the dependence of $k$ on the load is

$$
k(m)=k(m=0)\left(1-\alpha m^{2 / 3}\right),
$$

where $k(m=0)$ is the value determined from the unloaded bridge. The process is assumed to be activated with activation energy $\varepsilon_{A}$ and to have a vaporization rate proportional to the radius of the droplet,

$$
\frac{d m}{d t}=-\gamma_{T_{a}} \exp \left(\frac{-\varepsilon_{A}}{k_{B} T_{A}(t)}\right) m^{1 / 3},
$$

where $k_{B}$ is Boltzmann's constant $\left(1.381 \times 10^{-23} \mathrm{~J} / \mathrm{K}\right)$. Note that the temperature controlling the activation is not $\Delta T$ but the temperature of the analyte $T_{0}+\Delta T$. The sublimation (evaporation) enthalpies for TNT, RDX, and PETN are 498 $(402 \mathrm{~J} / \mathrm{g}), 588(424 \mathrm{~J} / \mathrm{g})$, and $476 \mathrm{~J} / \mathrm{g}(422 \mathrm{~J} / \mathrm{g})$, respectively. These enthalpies set the prefactor of the vaporized mass term in Eq. (4). The prefactor $\gamma_{T_{a}}$ and activation energy $\varepsilon_{A}$ are not readily available but can be selected to give reasonable agreement with the evolution of the response curves below the melting points of RDX and PETN. For TNT there is no indication of melting and we assume that the TNT is liquid or glass through the entire measurement. The response of TNT therefore illustrates the behavior of the bridge subject to vaporization only. There is a transient stage lasting $4 \mathrm{~ms}$ during which evaporative cooling is either suppressed by surface contamination or is counter balanced by weak exothermic reactions related to annihilation of defects. This is followed by a period of linearly increasing $\Delta T$ as the explosive evaporates at the rate appropriate to the ambient temperature. As $\Delta T$ grows heat flow controlled by $k$ contributes a quadratic term that reduces $\Delta T$ from linear growth. This is balanced by increasing activation of the vaporization process, which is controlled by the temperature of the bridge which initially grows slowly but then accelerates. Eventually the accelerating rate of evaporation causes $\Delta T$ to increase exponentially. The rapidly increasing vaporization results in a sudden depletion of the analyte. The peak in $\Delta T$ occurs when the cooling rate due to the vaporization of a small amount of remaining analyte matches the heat flowing in. Soon afterwards the analyte is essentially gone and according to Eq. (4) the temperature should increase back to $T_{0}$ with time constant $\tau$. Actually, for TNT in the last stage the observed behavior deviates from Eq. (4); the temperature increases faster than indicated by $\tau$. This can be seen in Fig. 7 where the temperature of the bridge with TNT increases to and surpasses the temperature of the reference bridge at a rate faster than predicted for the characteristic decay rate calculated for an unloaded bridge. We attribute this to an exothermic transformation turning on as $k_{B} T$ becomes comparable to the activation energy for deflagration $(621 \mathrm{~J} / \mathrm{g})$. The enthalpy of deflagration $(4560 \mathrm{~J} / \mathrm{g})$ is much higher than both the vaporization and melting enthalpies, therefore, even a small amount of deflagration can cause a return of $\Delta T$ to zero at a faster rate than the natural decay rate of the bridge. In fact, 


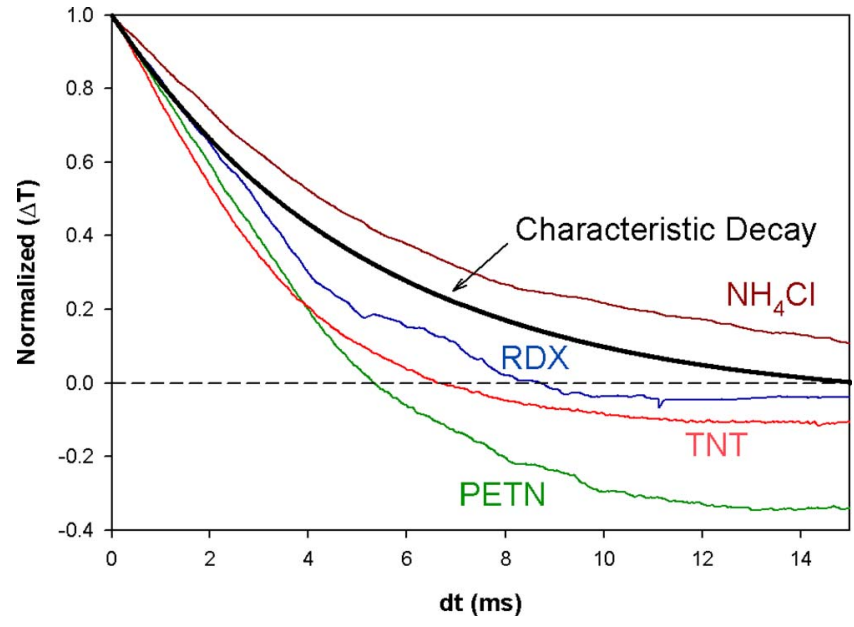

FIG. 7. (Color online) Comparison of the temperature decay rates. The characteristic decay is the expected temperature return rate for a bridge with no load and was calculated by the method described in Sec. IV. The nonexplosive $\mathrm{NH}_{4} \mathrm{Cl}$ has a rate slower than the characteristic rate due to thermal loading, while the explosives all show return rates faster than characteristic due to added heat from exothermic deflagration. The individual experimental responses are aligned in time and normalized to facilitate comparison to the calculated characteristic decay.

decay of $\Delta T$ faster or slower than indicated by the characteristic temperature decay time $\tau$ unambiguously indicates exothermic or endothermic reactions (reverse if $\Delta T$ is negative). This is illustrated in Fig. 7 where individual experimental responses are aligned in time and normalized to facilitate comparison to the calculated characteristic decay.

In RDX, PETN, and $\mathrm{NH}_{4} \mathrm{Cl}$ melting occurs. Melting results in a cooling of the sample proportional to the enthalpy of fusion that can be appended to Eq. (4),

$$
C \frac{d \Delta T}{d t}=-k \Delta T-\varepsilon_{V} \frac{d m}{d t}-\varepsilon_{F} \frac{d m_{\text {liquid }}}{d t} .
$$

Melting is assumed to take place instantaneously as a region of the analyte reaches the analyte melting point. Melting is initiated when the hottest part of the load reaches the melting point and continues until the coolest part of the load reached the melting point. This occurs over a fairly short period of time, therefore, the jump in $\Delta T$ due to melting can be estimated by $\Delta T_{\text {Melting }}=\varepsilon_{F} m / C$.

Another feature of the data is a noticeable shift in the response as the load is increased. For larger loads additional time (delay time) is required for the electric current to add the amount of Joule heat needed to overcome the cooling effect of vaporization and melting. The increase in heat capacity due to the specific heat of the load is found to have a very small effect on the delay. For explosives the major contributor to the delay is the vaporization cooling. This energy loss to vaporization cooling is approximately equal to $C \Delta T$ and the delay $\Delta t_{V}$ is given by this energy divided by the rate at which energy is accumulating in the bridge from Joule heating,

$$
\Delta t_{V}=C \Delta T\left(\frac{d C T_{0}}{d t}\right)^{-1}=\Delta T\left(\frac{d T_{0}}{d t}\right)^{-1} .
$$

The time delay due to melting can be estimated in a similar way,

$$
\Delta t_{\text {Melting }}=\varepsilon_{V} m_{\text {liquid }}\left(\frac{d T_{0}}{d t}\right)^{-1} .
$$

For the explosives the vaporization time delay is larger than the melting delay. For the $\mathrm{NH}_{4} \mathrm{Cl}$ the melting delay is larger. Adjusting the response curves to take these delays into effect gives alignment of response features corresponding to different loadings.

The simple analysis presented here allows thermodynamic quantities such as activation energies, enthalpies of fusion and evaporation, melting points, and deflagration to be identified in the response curves. Further pursuit of this approach could yield general guidelines for the detection of energetic molecules. The range of analyte interactions affects the thermodynamic properties as a function of analyte depth because the binding of the top layer is different from that in deeper levels closer to the bridge surface. Subtle effects of this type may reveal themselves with more detailed modeling.

By varying the voltage versus time function, different regimes can be highlighted. A slow increase in voltage emphasizes the evaporative regime. A rapidly increasing voltage will lead to deflagration prior to complete sublimation. We chose an intermediate time scale that displays the whole range of phenomena. A pulsed current gives an integrated response that is indicative of the analyte formation enthalpy.

\section{DISCUSSION}

Trace explosive vapor detection using thermally induced properties of adsorbed molecules offers a unique signal that can add specificity when used in conjunction with the partial selectivity achieved with array based systems of sensors with chemically selective layers. Since this detection method, which is free of any chemoselective layers, offers information that is orthogonal to the responses from chemoselective layer detection, the combination will provide better input for pattern recognition algorithms. Increased sensitivity beyond what is demonstrated will be possible by micromachining bridges with decreased thermal mass and maximum surface area. Perhaps the most important advantage of the method is that the detection process can be repeated continuously with the sensor regenerating to a pristine surface after each thermal cycle without resorting to chemical cleaning techniques. The technique could be extended for other chemical vapors with high vapor pressures. For example, the sensor bridges could be cooled below ambient temperature enabling enhanced adsorption or condensation of high vapor pressure analytes from the vapor phase.

The demonstrated sensitivity and selectivity of this approach offer new possibilities for a single sensor-based technique that does not use a chemoselective layer for sensing. This method may also provide a technique for the investigation of thermally induced properties of a wide range of materials far beyond what is possible by conventional techniques. These micromachined devices have the obvious advantage of requiring subnanogram samples, fast detection time, simple technique, and the potential to be low cost. 
Carrying out the temperature cycling in an inert atmosphere enables the investigation of the oxidative stability of analytes. It is also important to note that the sensing bridges could be heated with a gradient as high as a few millions of degrees/s, which will enable investigating the thermal properties of subnanogram quantities of materials under extremely high heating rates.

\section{CONCLUSION}

We described a technique for achieving chemical selectivity for adsorbed explosive molecules on a microfabricated silicon bridge using controlled heating of the bridge. The thermal response curves, which are due to a combination of melting, evaporation, and exothermic deflagration, are very unique to adsorbed explosives. We demonstrated that this technique can selectively detect explosives, providing a method for fast, direct detection with a LOD of 600 $\times 10^{-12} \mathrm{~g}$. The LOD can be further improved by optimization techniques. This method can be used in parallel with approaches based on chemically selective interfaces in a MEMS array providing orthogonal signals for pattern recognition. This technique has obvious advantages such as improved selectivity, sensitivity, low power operation, miniature size, and fast detection and regeneration.

\section{ACKNOWLEDGMENTS}

We thank Dr. Richard Lareau and Dr. Eric Houser for discussions on explosive detection. This research was supported in part by U.S. Department of Homeland Security and the Office of Naval Research. ORNL is managed by UT-Battelle, LLC for the U.S. Department of Energy under Contract No. DE-AC05-00OR22725.

${ }^{1}$ R. J. Colton and J. N. Russell, Science 299, 1324 (2003).

${ }^{2}$ S. F. Hallowell, Talanta 54, 447 (2001).

${ }^{3}$ A. Fainberg, Science 255, 1531 (1992).

${ }^{4}$ Counterterrorist Detection Techniques of Explosives, edited by J. Yinon (Elsevier B.V., New York, 2007).

${ }^{5}$ J. Yinon, Anal. Chem. 75, 98 A (2003)

${ }^{6}$ H. H. Hill and G. Simpson, Field Anal. Chem. Technol. 1, 119 (1997).

${ }^{7}$ S. K. Sharma, A. K. Misra, and B. Sharma, Spectrochim. Acta, Part A 61, 2404 (2005)

${ }^{8}$ L. A. Pinnaduwage, V. Boiadjiev, J. E. Hawk, and T. Thundat, Appl. Phys. Lett. 83, 1471 (2003).

${ }^{9}$ M. D. Hsieh and E. T. Zellers, Anal. Chem. 76, 1885 (2004).

${ }^{10}$ D. R. Walt, Anal. Chem. 77, (Issue 3), 45A (2005).

${ }^{11}$ C. J. Lu, J. Whiting, R. D. Sacks, and E. T. Zellers, Anal. Chem. 75, 1400 (2003).

${ }^{12}$ L. R. Senesac, P. Dutta, P. G. Datskos, and M. J. Sepaniak, Anal. Chim. Acta 558, 94 (2006).

${ }^{13}$ P. Dutta, L. R. Senesac, N. V. Lavrik, P. G. Datskos, and M. J. Sepaniak, Sens. Lett. 2, 238 (2004).

${ }^{14}$ D. Yi, A. Greve, J. H. Hales, L. R. Senesac, Z. J. Davis, D. M. Nicholson, A. Boisen, and T. Thundat, Appl. Phys. Lett. 93, 154102 (2008).

${ }^{15}$ J. Lee, T. Beechem, T. L. Wright, B. A. Nelson, S. Graham, and W. P. King, J. Microelectromech. Syst. 15, 1644 (2006).

${ }^{16}$ L. A. Pinnaduwage, A. Wig, D. L. Hedden, A. Gehl, D. Yi, T. Thundat, and R. T. Lareau, J. Appl. Phys. 95, 5871 (2004).

${ }^{17}$ L. A. Pinnaduwage, A. Gehl, D. L. Hedden, G. Muralidharan, T. Thundat, R. T. Lareau, T. Sulchek, L. Manning, B. Rogers, M. Jones, and J. D. Adams, Nature (London) 425, 474 (2003).

${ }^{18}$ L. A. Pinnaduwage, D. Yi, F. Tian, T. Thundat, and R. T. Lareau, Langmuir 20, 2690 (2004).

${ }^{19}$ R. I. Masel, Principles of Adsorption and Reaction on Solid Surfaces (Wiley, New York, 1996), p. 509.

${ }^{20}$ R. Mu, A. Ueda, M. H. Wu, Y. S. Tung, D. O. Henderson, R. T. Chamberlain, W. Curby, and A. Mercado, J. Phys. Chem. B 104, 105 (2000). 